\title{
The Use of Functional Music in the Exploration of Middle and Long Distance Running
}

\author{
Zhang Yiwen \\ Jiangxi normal university, No. 99, Ziyang Avenue, Nanchang County, Nanchang, Jiangxi, China \\ 1036988432@qq.com
}

Keywords: Functional music, Middle and long distance running training

\begin{abstract}
Sports is exercise. Many scholars also call it the education of the body, mainly the external manifestation and then the internal influence. Music, on the other hand, is an art that has the effect of regulating human emotions, mainly on the inner spiritual level, through external expressions, and thus affecting the inner. At first glance, music and sports seem to have no connection; after exploring, it is found that music and sports actually have inextricably linked. Sports is a kind of physical movement. Music is an emotional activity, that is, psychological movement. Human body and psychology constitute a complete person. This article explores the connection between functional music and middle and long distance running training.
\end{abstract}

\section{The connection between functional music and sports training}

In the process of evolution and advancement of sports, sports are constantly colliding and intersecting with other fields. The development of modern sports is also inseparable from the support and improvement of various disciplines. Music and sports are also inextricably linked. The most closely related of them is all kinds of dances. Music is the melody and sports are the expression. In dance, music and sports have been integrated.

Functional music refers to music with a certain use value, which is different from purely artistic music that is generally only for appreciation. Further subdividing refers to music with social practical benefits (physical or psychological). The actual benefits of functional music are determined by several factors, including the user's motivation, purpose, circumstances, or role. From the perspective of the practicality of functional music, its most important psychological effect is the impact on human basic emotions, and some physiological functions. Functional music can be divided into three types, namely, mobilizing music, relaxing music, and calm music. It corresponds to the preparation part, the basic part and the end part of a complete exercise training, that is, preparation for mobilization, formal training and end of relaxation.

\section{The special role of functional music in middle and long distance running training}

The middle and long run is a physical endurance endurance project in sports and is a typical periodic exercise. To achieve excellent athletic performance, it is inseparable from the physical fitness, superb technology and good performance in the competition. To improve these elements, it 
is inseparable from scientific training. The middle and long-distance running project has a long history of development. The training methods of various physical fitness, skills and mental abilities are very mature and difficult to break through the support of modern science. How to use various training methods under the existing conditions To improve or break through has become a way of thinking worth exploring. In recent years, there have been scholars and coaches who are trying to use functional music in other projects other than dance gymnastics. The connection between functional music and sports training is also closer. After revealing the characteristics of functional music and middle-distance running training, and the intersection of the two, the feasibility of functional music in mid-long-distance running training can be analyzed and discussed.

\subsection{The regulation of physiological functions in functional long-distance running training}

The performance of the middle and long-distance running depends on the speed of the athletes running in the whole process; and if the athlete wants to create better athletic performance, he must use the effective skills to maximize the physical fitness. In the process of running on the way, the combination of the athlete's stride frequency and breathing, the control of the excitability of the working muscle group, that is, the rhythm of running, is the key to the technical application. In the process of training, the training of athletes with good rhythm with functional music can make the athletes have more stable pace and breathing rhythm in the whole running process, and consciously in each running, experience the work. The excitement and inhibition of the muscle group reduces the energy consumption of running, reduces the fatigue in running, and delays the appearance of physiological polar regions.

In addition to the melody that regulates people's emotions, the most distinctive feature of functional music is the distinct musical rhythm. By using software to present the rhythm of music in the form of a waveform diagram, it can be found that in a piece of music, the rhythm of the music is stable and the tempo is noticeably tempered, which is exactly the steady pace required for the middle and long distance running. The rhythm coincides with the alternating appearance of excitatory inhibition of running muscles. Choosing the right music can better cultivate the rhythm of the athletes and reduce the energy consumption of the working muscles.

In the article on gymnastics and music, Zhang Chunde pointed out that under the influence of music, athletes have a large difference in lung ventilation and oxygen uptake during preparation activities. In the absence of music accompaniment, the athletes do"stretching"with a lung ventilation of 5.97L per minute and an oxygen uptake of $365 \mathrm{ml}$ per minute. The lung ventilation of the athlete who performed the same action with music accompaniment was $8.23 \mathrm{~L}$ per minute and the oxygen uptake was $433 \mathrm{ml}$ per minute. It can be seen that in a preparatory activity, whether or not music accompaniment can play such a significant effect on the athlete's breathing and oxygen uptake, then in the longer and longer middle and long distance running, the use of music will be able to play a larger role. effect. In the article on the function and function of music in sports, Chen Fang pointed out that under the influence of music, the pulse, blood pressure and breathing of athletes will also change accordingly. It can give athletes a stronger desire to exercise, thus creating the excitement needed for excellent athletic performance.

The climax of music that most inspires people's emotions does not appear at the beginning, but has a transition. After grasping the time law of the music climax, the time of the music climax point is arranged in the fatigue period before the athlete's physiological pole. It is necessary to delay the appearance time of the athlete's physiological poles and improve the running results. 


\subsection{The adjustment effect of functional music on psychological state in middle and long distance running training}

Among the influencing factors of sports performance, it also includes the performance of athletes' training performance. How to play the superb training level in the game, it is very important for the performance of the game. Training and competition are two different situations. In the game, athletes will inevitably be nervous and unstable. In order to restore the athletes' good mentality in training, some methods and methods, such as the idea training method and the induction training method, are needed. Before the game, let the athletes listen to the music often used in training, which is equivalent to using an effective stimulus to guide the athletes to establish a good psychological state of the game.

\subsection{The promotion of functional music to relaxation and recovery after long-distance running training}

A complete training and competition, after the end must be arranged to relax and recover, in the regular massage stretching process, add functional music - play soothing and calm music, can better eliminate the athlete's fatigue. Playing the right music can accelerate the regression of fatigue from both physiological and psychological functions. From the physiological function, the soothing rhythm, speed and beautiful melody can reduce the athlete's heart rate blood pressure and excitability, relax the athlete's muscles; from the psychological function, can bring joy to the athletes, eliminate the training competition Tension, mental fatigue caused by long boring, intense training. Therefore, functional music, as a supplement to the conventional relaxation and recovery, has a good promotion effect on relaxation and recovery after the middle and long-distance running training.

\section{Conclusions}

(1) After analyzing the middle and long-distance running project and the characteristics of functional music, it is found that in the training of middle and long distance running, reasonable sports functional music can play a certain role in promoting the training effect and the performance of the competition.

(2) Using the melody and rhythm of the functional sound, can train the rhythm of the athletes to run, reduce the energy consumption of the working muscles; enable the athletes to generate stronger sports desires, thus creating the excitement required for excellent sports performance; The appearance time of athletes' physiology poles improves the performance of running.

(3) The use of functional music in training and competition can induce athletes to resume training and establish a good psychological state of the game.

(4) In the process of relaxation, functional music can make the athlete's psychological function and physiological function get better relaxation and recovery effect.

\section{References}

[1] Fei Yuguang, Dong Liying, et al. Experimental research on the use of functional music in middle and long distance running training [J] Heilongjiang: Journal of Harbin Institute of Physical Education, 2007 (3): 102-104.

[2] Yongming. The experiment and application of music in the middle and long distance running teaching[J]. Heilongjiang: Journal of Mudanjiang Teachers College,2006(3)46-47.

[3] Chen Xiaoguang, Xu Liang et al. Study on the elimination of exercise fatigue in physical exercise by negative oxygen ion plus music regulation[J], Henan: Journal of Pingyuan University, 2003(5)87-88.

[4] Fan Xiaoyan, Gong Bin et al. Analysis of psychological factors in the combination of music and sports[J]. Henan: 
Journal of PLA Institute of Physical Education,2001(3)49-50.

[5] Gong Bin, Bao Weiping. Analysis of the Influence of Musical Rhythm on Sports[J]. Inner Mongolia: Yinshan Academic Journal, 2001(6) 54-56. 